\title{
Characterization of different biodegradable scaffolds in tissue engineering
}

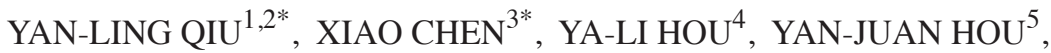 \\ SONG-BO TIAN ${ }^{6}$, YU-HE CHEN ${ }^{1,2}$, LI YU ${ }^{1,2}$, MIN-HAI NIE ${ }^{1,2}$ and XU-QIAN LIU ${ }^{1,2}$ \\ ${ }^{1}$ Department of Periodontics and Oral Mucosa, Affiliated Stomatology Hospital; ${ }^{2}$ Laboratory of Oral and \\ Maxillofacial Reconstruction and Regeneration, Southwest Medical University, Luzhou, Sichuan 646000; \\ ${ }^{3}$ Department of Orthodontics, Mianyang Stomatological Hospital, Mianyang, Sichuan 621000; ${ }^{4}$ Department of Oral Pathology, \\ College and Hospital of Stomatology, Hebei Medical University and The Key Laboratory of Stomatology, Shijiazhuang, \\ Hebei 050000; ${ }^{5}$ Department of Nephrology, Second Hospital, Shanxi Medical University, Taiyuan, Shanxi 030001; \\ ${ }^{6}$ Department of Oral Medicine, Second Hospital of Hebei Medical University, Shijiazhuang, Hebei 050000, P.R. China
}

Received May 15, 2018; Accepted December 19, 2018

DOI: $10.3892 / \mathrm{mmr} .2019 .10066$

\begin{abstract}
The aim of the present study was to compare the characteristics of acellular dermal matrix (ADM), small intestinal submucosa (SIS) and Bio-Gide scaffolds with acellular vascular matrix (ACVM)-0.25\% human-like collagen I (HLC-I) scaffold in tissue engineering blood vessels. The ACVM-0.25\% HLC-I scaffold was prepared and compared with ADM, SIS and Bio-Gide scaffolds via hematoxylin and eosin (H\&E) staining, Masson staining and scanning electron microscope (SEM) observations. Primary human gingival fibroblasts (HGFs) were cultured and identified. Then, the experiment was established via the seeding of HGFs on different scaffolds for 1, 4 and 7 days. The compatibility of four different scaffolds with HGFs was evaluated by H\&E staining, SEM observation and Cell Counting Kit-8 assay. Then, a series of experiments were conducted to evaluate water absorption capacities, mechanical abilities, the ultra-microstructure and the cytotoxicity of the four scaffolds. The ACVM-0.25\% HLC-I scaffold was revealed to exhibit the best cell proliferation and good cell architecture. ADM and Bio-Gide scaffolds exhibited good mechanical stability but cell proliferation was reduced when compared with the ACVM-0.25\% HLC-I scaffold. In addition, SIS scaffolds exhibited the worst cell proliferation.
\end{abstract}

Correspondence to: Professor Xu-Qian Liu or Professor Min-Hai Nie, Department of Periodontics and Oral Mucosa, Affiliated Stomatology Hospital, Southwest Medical University, 2 Jiang Yang Nan Road, Jiangyang, Luzhou, Sichuan 646000, P.R. China

E-mail: liuxuqianwork@163.com

E-mail: nieminhai@126.com

*Contributed equally

Key words: scaffold, acellular vascular matrix, small intestinal submucosa
The ACVM-0.25\% HLC-I scaffold exhibited the best water absorption, followed by the SIS and Bio-Gide scaffolds, and then the ADM scaffold. In conclusion, the ACVM-0.25\% HLC-I scaffold has good mechanical properties as a tissue engineering scaffold and the present results suggest that it has better biological characterization when compared with other scaffold types.

\section{Introduction}

The three essential elements of tissue engineering are seed cells, scaffold materials and cytokines (1-3). The selection of tissue engineering scaffold material is directly associated with the effect of the material implanted in the animal or human body. Most importantly, the scaffold materials should have good biocompatibility (4-6). Scaffold materials can provide a good adsorption interface for the adhesion of seed cells, in order to facilitate cell proliferation. In addition, the scaffold material must have a certain level of biomechanical strength $(7,8)$ and maintain a controlled degradation rate once implanted into the animal or human body. The material should have no cytotoxic, immunogenic, tumorigenic or teratogenic effects on animals or humans. In addition, the scaffold materials are required to integrate cytokines and to activate the expression of various specific genes, Runt-related transcription factor 2, osteocalcin (9), peroxisome proliferator-activated receptor- $\gamma$ (10) and $\alpha$-smooth muscle actin (11) in order to maintain the normal phenotype of the seed cells on the scaffold surface (12).

Classes of scaffold materials include natural biological scaffold materials, synthetic biodegradable polymer scaffolds materials (13-15), composite scaffold materials (16-18) and nano-scaffold materials (16-18). Natural biological scaffolds have good biocompatibility but poor mechanical properties (19). Synthetic biodegradable polymer scaffolds tend to have strong mechanical properties (20), but lack good cellular compatibility. Nano-scaffold materials have been widely studied in recent years, but are expensive to produce (21). 
Currently, the most widely used scaffold materials are composite scaffold materials. These benefit from the good cellular compatibility of natural biological scaffolds and the strong mechanical properties of synthetic biodegradable polymer scaffolds (22).

In our previous studies, human-like collagen I (HLC-I) (23) was combined with the acellular vascular matrix $(\mathrm{ACVM})(23,24)$ to construct the ACVM-0.25\% HLC-I scaffold material (25). This process involved combining a polymer material with natural degenerative biomaterials to construct a composite tissue engineering scaffold material. This material supports the growth of seed cells with a three-dimensional ultrastructure of protein space to promote the formation of functional tissue, but it also possesses compressive mechanical properties required for vascular tissue engineering (25). A previous study demonstrated that vascular endothelial cells and vascular smooth muscle cells are induced and differentiated by human gingival fibroblasts (HGFs) (25). Combining these cells with ACVM-0.25\% HLC-I scaffold materials supported successful tissue engineering of vessels that possessed the morphological and immune characteristics of vascular tissue. The structure of the tissue-engineered vascular tissue was intact following 9 weeks in nude mice (25). Thus, the aforementioned results demonstrated the feasibility of ACVM-0.25\% HLC-I scaffold material for tissue engineering.

The present study compares the biological characteristics of different ACVM-0.25\% HLC-I scaffold materials with Acellular Dermal Matrix (ADM) $(26,27)$, Small Intestinal Submucosa (SIS) $(28,29)$ and Bio-Gide scaffolds in tissue engineering. The aim of the present study was to confirm the advantages of ACVM-0.25\% HLC-I composite scaffolds and lay a foundation for the research of tissue engineering scaffold materials.

\section{Materials and methods}

\section{Preparation of vascular scaffolds}

Preparation of ACVM-HLC-I scaffolds. Female 12-week-old New Zealand white rabbits, weighing 1.8-2.0 kg, were provided by the Animal Center of Hebei Medical University (Shijiazhuang, China; license no. SCXK 2013-1-003; certification no. 1505098). The rabbits were housed in plastic cage (1 rabbit per cage) and were allowed free access to food and water. The housing conditions were maintained at temperature of $25^{\circ} \mathrm{C}$, relative humidity of $40-70 \%$, under a 12 -h light/dark cycle. Animal experiments were approved by the Ethics Committee of Southwest Medical University (Sichuan, China). ACVM-HLC-I scaffolds were prepared as described previously (23). Briefly, the blood vessels of New Zealand white rabbits were isolated and washed in saline solution eight times. Sections of blood vessel $2 \mathrm{~cm}$ in length were immersed in PBS containing $1 \%$ benzalkonium bromide (Lircon) for $1 \mathrm{~h}$ at room temperature and then in PBS for 5 min. The vessels were then placed in a liquid mixture with $1 \%$ trypsin and $0.01 \%$ EDTA for $24 \mathrm{~h}$ at $37^{\circ} \mathrm{C}$ and $5 \% \mathrm{CO}_{2}$. Following washing with PBS for 5 min, samples were incubated in PBS containing $1 \%$ Triton-X-100 for $72 \mathrm{~h}$ at room temperature. Finally, blood vessels were immersed in sterile PBS solution at $4^{\circ} \mathrm{C}$ for subsequent experiments.

Then, the ACVM scaffolds were prepared as follows: The bio-activation of ACVM-HLC-I was performed by firstly using a solution of acrylic acid to reduce the dissolved oxygen on the surface of the ACVM scaffolds. The scaffolds were then exposed to UV radiation for $30 \mathrm{~min}$ and placed in a Petri dish. The scaffolds were washed with distilled water to remove the excess homo-polymer and placed to dry in a vacuum desiccator. Immobilization of HLC-I was performed once the surface of the scaffolds was modified with acrylic acid. The ACVM-acrylic acid scaffolds were immersed at $4^{\circ} \mathrm{C}$ for $1 \mathrm{~h}$ in PBS, which also contained $5 \mathrm{mg} / \mathrm{ml}$ water-soluble carbodiimide in order to activate the carboxyl groups on the surface of the ACVM scaffolds. Secondly, various concentrations of HLC-I $(0.10,0.25,0.50,0.75$ and $1.00 \mathrm{mg} / \mathrm{ml})$ were added to combine with the surface of the ACVM for $5 \mathrm{~h}$ at room temperature. Thirdly, the scaffolds were washed with PBS at room temperature for $1 \mathrm{~h}$ to remove the excess HLC-I that was physically adsorbed on the surface of the scaffold. Finally, ACVM-HLC-I scaffolds were dried under reduced pressure and stored at $4^{\circ} \mathrm{C}$.

Preparation of SIS, ADM and Bio-Gide scaffolds. Serosa and muscle of the jejunum were removed using gauze. Then, the jejunum was immersed in PBS that contained $1 \%$ penicillin and $1 \%$ streptomycin for $3 \mathrm{~h}$ at room temperature. Trypsin $(0.25 \%)$ was added to the jejunum for $24 \mathrm{~h}$ at room temperature and then it was immersed in $0.5 \%$ SDS for $24 \mathrm{~h}$. The jejunum was washed with distilled water twice. Finally, the jejunum was sterilized in sterile water containing $20 \%$ ethanol and $0.1 \%$ peracetic acid for $10 \mathrm{~h}$ at room temperature, then freeze-dried for use. The ADM scaffold was purchased from Beijing Qingyuanweiye Bio-Tissue Engineering, Co., Ltd., (Beijing, China), and the Bio-Gide scaffold was purchased from Geistlich Pharma AG (Wolhusen, Switzerland).

Cell isolation and culture of primary cells. HGFs were used for cell studies. HGFs were isolated from healthy gingival tissue, which was harvested from clinical donors undergoing mandibular third impact molar extraction as described previously (30). The acquisition of normal human gingival tissues from patients was approved by the Hospital of Stomatology at Hebei Medical University (Hebei, China), and written informed consent was obtained from all patients. Following the removal of the epithelial layer, human gingival tissues were cut into $1 \times 1 \mathrm{~mm}^{2}$ pieces and placed in low-glucose Dulbecco's modified Eagle medium (L-DMEM; Hyclone; GE Healthcare Life Sciences, Logan, UT, USA) containing $10 \%$ fetal bovine serum (Gibco; Thermo Fisher Scientific, Inc., Waltham, MA, USA), $100 \mathrm{U} / \mathrm{ml}$ penicillin (Shenzhen Huayao Nanfang Pharmaceutical Co., Ltd., Shenzhen, China), and 100 U/ml streptomycin (Shenzhen Huayao Nanfang Pharmaceutical Co., Ltd.). Cells at the second or the third passage were from 6 different donors in the present study.

Immunofluorescence staining of HGFs. HGFs were immersed in acetone and washed with PBS. Then, HGFs were fixed in $4 \%$ paraformaldehyde at $4^{\circ} \mathrm{C}$ overnight and washed with PBS three times. The following primary antibodies were used for cell incubation at $37^{\circ} \mathrm{C}$ for $2 \mathrm{~h}$ : Vimentin (1:100; ZM-0260; Beijing Zhongshan Golden Bridge Biotechnology Co.,

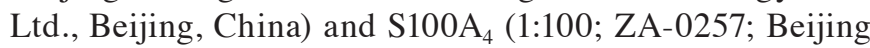
Zhongshan Golden Bridge Biotechnology Co., Ltd.). $\mathrm{H}_{2} \mathrm{O}_{2}$ (3\%) was added to blocking protease for $20 \mathrm{~min}$ at room 
temperature and then $30 \mu \mathrm{l}$ sheep serum blocking buffer (ZC-02125; Beijing Zhongshan Golden Bridge Biotechnology Co., Ltd.) was added for $1 \mathrm{~h}$ at room temperature. Following three washes with PBS, the cells were incubated with the following secondary antibodies at $37^{\circ} \mathrm{C}$ for $1 \mathrm{~h}$ : Fluorescein isothiocyanate-conjugated goat anti-mouse immunoglobulin G (IgG; 1:100, ZF-0312; Beijing Zhongshan Golden Bridge Biotechnology Co., Ltd.) and rhodamine-conjugated goat anti-rabbit IgG (1:100; ZF-0316; Beijing Zhongshan Golden Bridge Biotechnology Co., Ltd.). Cell nuclei were stained with 4',6-diamidino-2-phenylindole for $10 \mathrm{~min}$ at room temperature. Finally, the cells were photographed under a laser confocal microscope (Olympus Corporation, Tokyo, Japan).

Optimal concentration of HLC-I crosslinking with ACVM scaffolds measured by MTT. ACVM scaffolds with various concentrations of HLC-I (ACVM-HLC-I scaffolds) were cut into smaller blocks of $10.0 \mathrm{~mm}$ diameter. Scaffolds were placed in a 24-well culture plate. A volume of $100 \mu \mathrm{l}$ L-DMEM containing $1 \times 10^{5}$ HGFs was placed on each scaffold. The seeded scaffolds were placed in a $37^{\circ} \mathrm{C}$ incubator for $2 \mathrm{~h}$ and then transferred to another 24-well plate, following which $2 \mathrm{ml}$ culture medium was added to each well. The culture medium was replaced every 2 days. The number of cells of each scaffold were tested by MTT assay following culture for 1, 4 and 7 days.

For the MTT assay, HGFs on each scaffold were incubated with L-DMEM for $12 \mathrm{~h}$ at $37^{\circ} \mathrm{C}$. Then, $10 \mu \mathrm{l}$ of $5 \mathrm{mg} / \mathrm{ml}$ MTT (Beijing Zhongshan Golden Bridge Biotechnology Co., Ltd.) was added to each well. A well without cells was used as the blank control. The plate was incubated at $37^{\circ} \mathrm{C}$ for $3.5 \mathrm{~h}$. Then, $100 \mu \mathrm{l}$ DMSO was added to each well and incubated at room temperature for $10 \mathrm{~min}$. Absorbance at $490 \mathrm{~nm}$ was measured by microplate reader to calculate the concentration of cells on ACVM-HLC-I scaffolds.

\section{Observation analysis of the vascular scaffolds}

Histological analysis. Sections of scaffold $(5 \mathrm{~cm})$ were mounted on light microscope slides and Masson Trichrome staining (HT15; Sigma-Aldrich; Merck KGaA, Darmstadt, Germany) was performed. The slides were stained with Masson complex staining for $5 \mathrm{~min}$ at room temperature and washed with $0.2 \%$ acetic acid water. Then, the slides were immersed in $5 \%$ phosphotunfstic acid for $5 \mathrm{~min}$ and washed with $0.2 \%$ acetic acid water twice. Following deparaffinization, the slides were stained with Bouin's solution at $56^{\circ} \mathrm{C}$ for $15 \mathrm{~min}$. Hematoxylin and eosin (H\&E) staining was used to investigate the basic structure of ACVM-0.25\% HLC-I scaffold compared with SIS, ADM and Bio-Gide scaffolds, and to observe the four different biodegradable scaffolds following seeding with HGFs. After dewaxing, the slides were stained with hematoxylin for $5 \mathrm{~min}$ at room temperature, following by washing with water for $2 \mathrm{~min}$. Then, the slides were stained with eosin for $30 \mathrm{sec}$, and next washed with water for $2 \mathrm{~min}$. Masson staining was performed to evaluate the fiber composition of the ACVM-0.25\% HLC-I, SIS, ADM and Bio-Gide scaffolds.

Water absorption capacities. The water absorption capacities of the four scaffolds were determined by the degree of swelling in PBS at room temperature. A known weight of the scaffold material was placed in PBS for $12 \mathrm{~h}$. The wet weight of the scaffold was determined as the weight of the scaffold after blotting the surface with filter paper to remove the extra PBS. The percentage of water absorption of the scaffolds was calculated using the following equation (23): $\mathrm{W}_{1}-\mathrm{W}_{0} / \mathrm{W}_{0} \times 100 \%$, where $W_{1}$ represents the wet weight of the scaffolds after $24 \mathrm{~h}$ and $\mathrm{W}_{0}$ is the initial weight of the scaffolds.

Measurement of scaffold mechanical ability. The stress-time and stress-strain curves of the scaffolds were tested using a universal material testing machine (Zwick Roell Z020; Zwick Roell Group, Ulm, Germany). The scaffolds were divided into four groups: ACVM-0.25\% HLC-I scaffold, ADM scaffold, Bio-Gide scaffold and SIS scaffold groups. During the whole experiment, PBS was used to keep the samples moist. All samples were pre-adjusted prior to testing. The length of the specimen was $1 \mathrm{~cm}$ and it was stretched at a speed of $10 \mathrm{~mm} / \mathrm{min}$ until it snapped. The stress and strain were subsequently recorded. Stress is the mechanical state describing every direction of every point within an object. It is described as the force per unit area. Strain is the amount of change in the shape of a material under external force. The stress-strain curves were plotted on the basis of stress as the ordinate and strain as the abscissa. The breaking strength and elongation at break were also calculated. Breaking strength = maximum stress when the material breaks / cross-sectional area. Elongation at break $(\%)=$ displacement value of material when it breaks/original length of material.

Scanning electron microscope (SEM) imaging. The samples were fixed in a metal and coated with a thin layer of gold, using an Edwards EXC 120 Turbo Pump Controller. A SEM (JSM-5310; JEOL, Ltd., Tokyo, Japan) was used to investigate the surface morphology of the four scaffolds.

Observation analysis of the vascular scaffolds following seeding with HGFs

Histological analysis. Scaffold sections $(5 \mathrm{~cm})$ were mounted on light microscope slides and Masson Trichrome staining was performed at room temperature. The slides were stained with Masson complex staining for $5 \mathrm{~min}$ and after washed with $0.2 \%$ acetic acid water. Then, the slides were immersed in $5 \%$ phosphotunfstic acid for $5 \mathrm{~min}$ and washed with $0.2 \%$ acetic acid water twice. Following dewaxing, the slides were stained with Bouin's solution at $56^{\circ} \mathrm{C}$ for $15 \mathrm{~min}$. $\mathrm{H} \& \mathrm{E}$ staining was used to evaluate ACVM-0.25\% HLC-I, SIS, ADM and Bio-Gide scaffolds after seeding with HGFs. Tissue samples for histology were fixed in $4 \%$ paraformaldehyde at $4^{\circ} \mathrm{C}$ for $24 \mathrm{~h}$ and embedded in paraffin. After dewaxing, the slides were stained with hematoxylin for $5 \mathrm{~min}$ at room temperature, following by washing with water for $2 \mathrm{~min}$. Then, the slides were stained with eosin for $30 \mathrm{sec}$, then with water for $2 \mathrm{~min}$.

SEM imaging. The surface morphology of HGFs on the four different biodegradable scaffolds was observed using an JSM-6320F SEM (JEOL, Ltd.), as aforementioned.

Growth kinetics of HGFs on scaffolds. Cell Counting Kit-8 (CCK-8) was used to evaluate the growth kinetics of HGFs on 

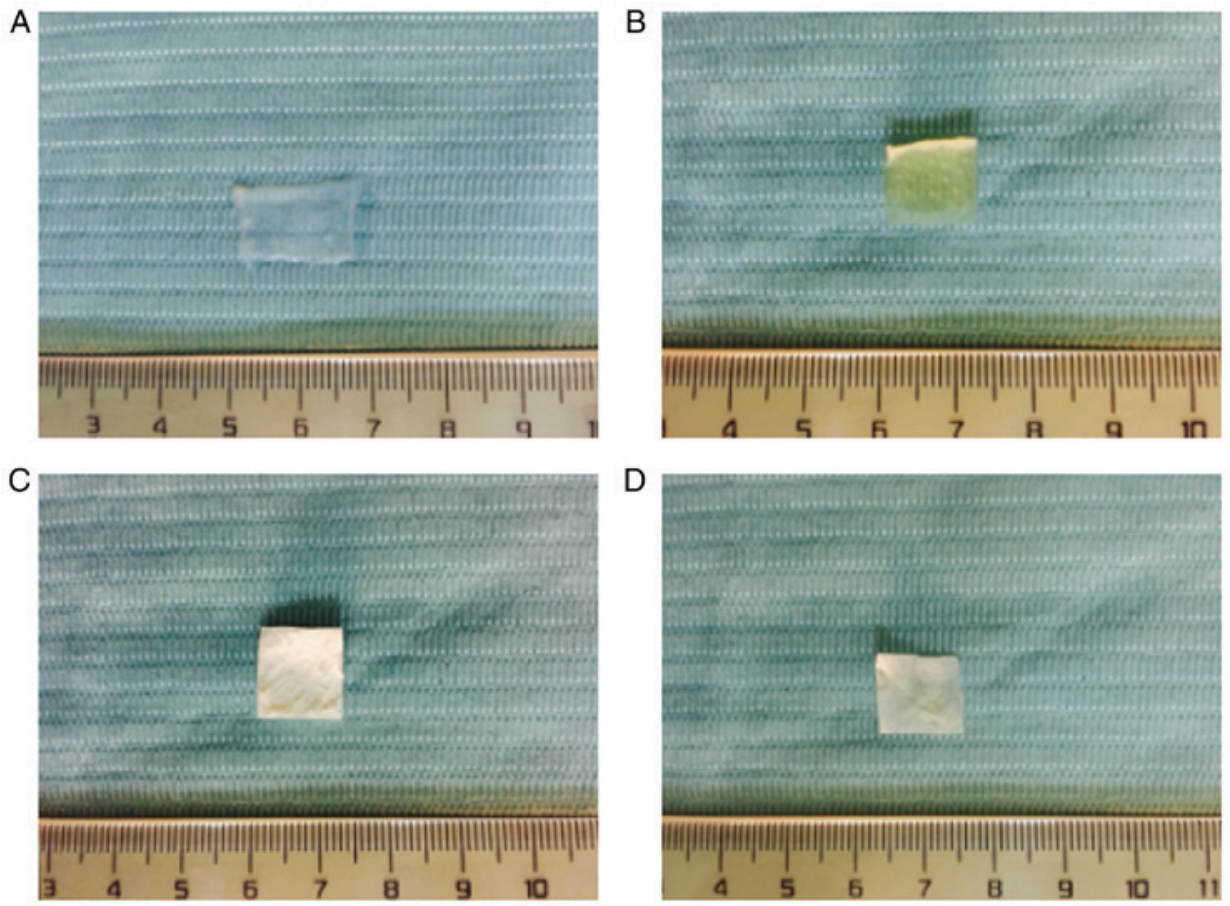

Figure 1. Observation of scaffold materials. (A) ACVM-HLC-I, (B) ADM, (C) Bio-Gide and (D) SIS. ACVM-HLC-I scaffold presented as an ivory-white, translucent and non-elastic vascular wall with a folded and damaged lumen. ADM presented with an ivory-white, translucent, honeycomb structure with a rough surface. Bio-Gide was pale yellow-white, with translucent rectangular patches and a smooth surface. SIS presented as a pale ivory, translucent membrane. ACVM-HLC-I, acellular vascular matrix-human-like collagen I; ADM, acellular dermal matrix; SIS, small intestinal submucosa.
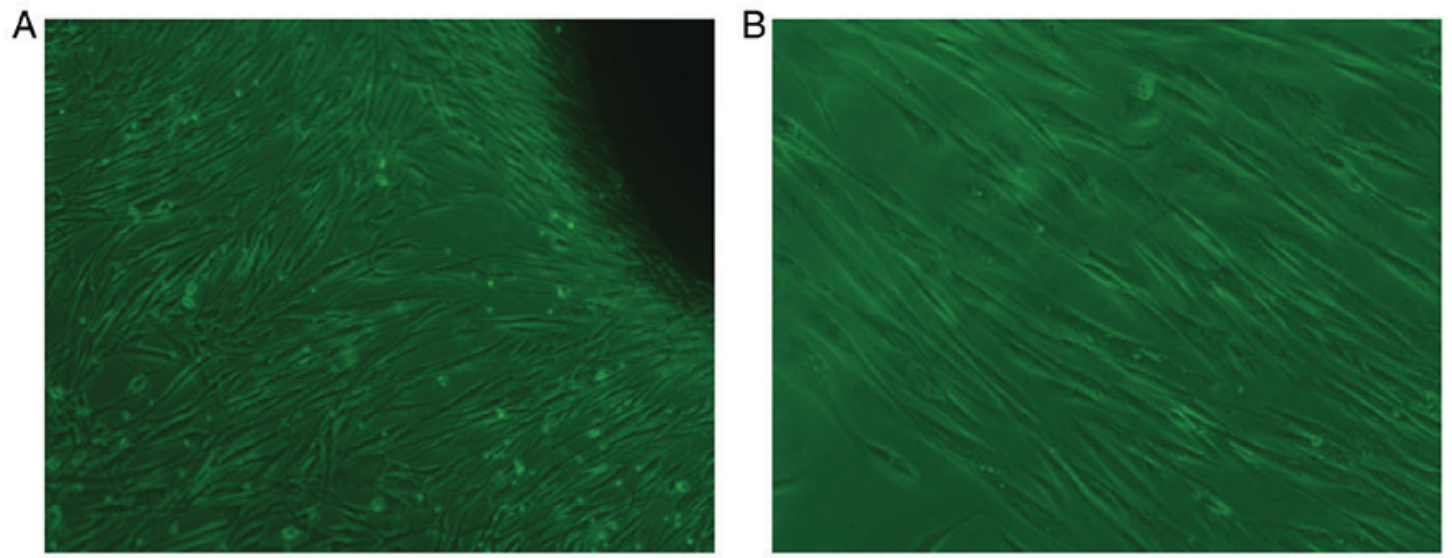

Figure 2. Culture of HGFs. (A) Day 7 of primary culture of HGFs (inverted microscope; magnification, x400). (B) Day 15 of second generation of HGFs (inverted microscope; magnification, $\mathrm{x} 400$ ). The primary culture of HGFs exhibited clear outlines and large, spherical or elliptic nuclei. Second generation cells were spindle-shaped and fibroblast-like. HGF, human gingival fibroblast.

the four scaffolds. A total of $2 \times 10^{3} \mathrm{HGFs} /$ well were seeded on the four scaffolds inoculated in 96-well microplates. CCK-8 solution $(10 \mu \mathrm{l})$ was added to each well and incubated at $37^{\circ} \mathrm{C}$ for $1 \mathrm{~h}$. Subsequently, the absorbance of each well was measured by a microplate reader at $450 \mathrm{~nm}$ on days 1,4 and 7 after cell seeding. The absorbance values at different time points were used to construct a growth curve to indicate the growth kinetics of HGFs on the four scaffolds.

Changes in the diameter of the scaffolds following $\mathrm{HGF}$ seeding. A vernier caliper was used to measure the diameter of the ACVM-0.25\% HLC-I, ADM, Bio-Gide and SIS scaffolds prior to seeding with HGFs. The diameters of all scaffolds were measured to be $10 \mathrm{~mm}$. The density of $2 \times 10^{5} \mathrm{HGFs}$ were placed on the surface of each scaffold and cultured in a $37^{\circ} \mathrm{C}$ incubator for $48 \mathrm{~h}$. Then, the scaffolds were removed and the culture fluid was aspirated with sterile filter paper. The diameter of each scaffold was measured using a vernier caliper following compounding of HGFs.

Statistical analysis. Statistical analysis was performed by using SPSS software (v. 21.0; IBM Corp., Armonk, NY, USA). The measurement data are presented as the mean \pm standard deviation and were analyzed by one-way analysis of variance, followed by a Student-Newman-Keuls multiple comparison test. $\mathrm{P}<0.05$ was considered to indicate a statistically significant difference. 

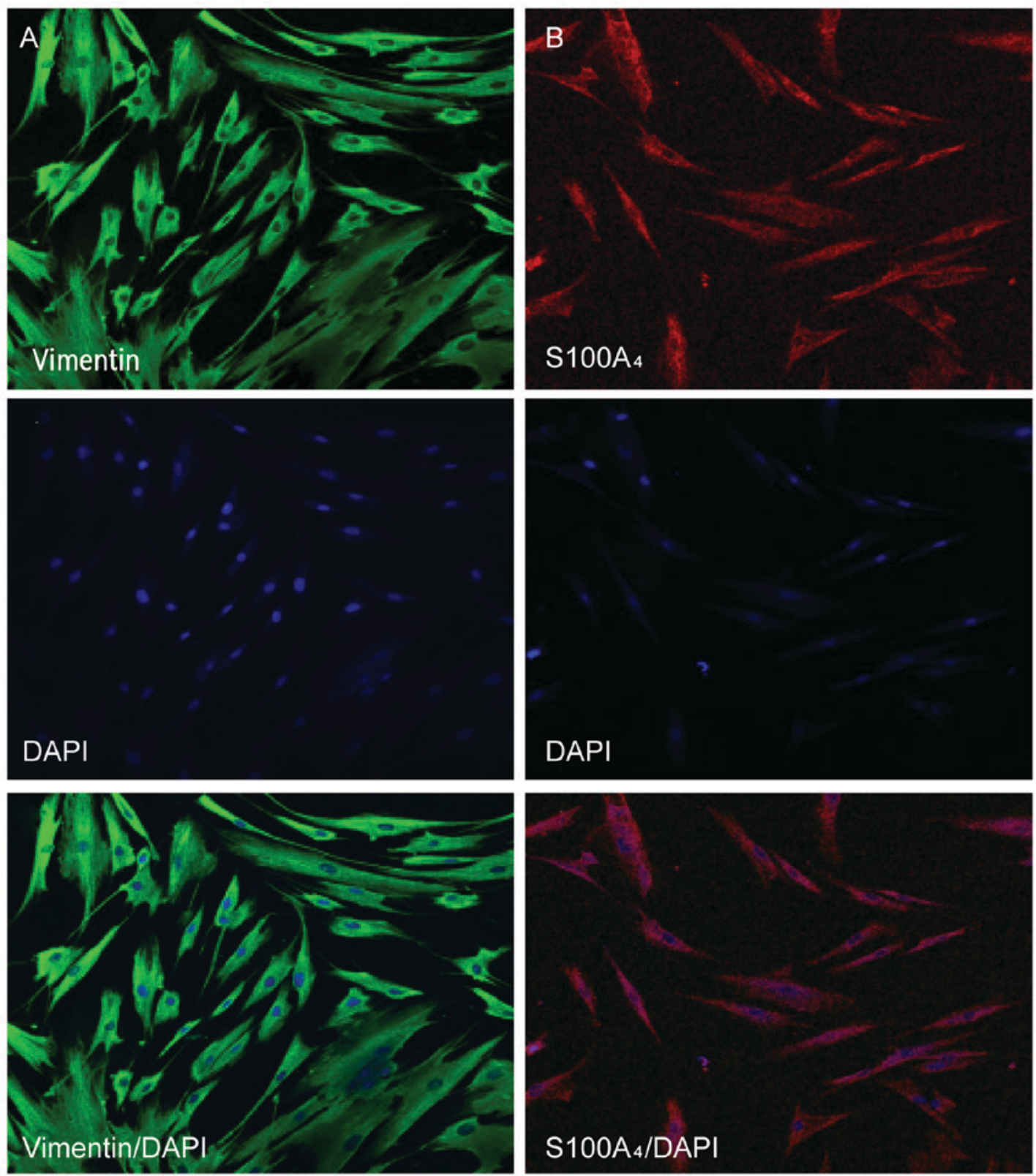

Figure 3. Immunofluorescent staining of HGFs. (A) Cytoplasm of HGFs with positive staining for vimentin (fluorescein isothiocyanate; magnification, $\mathrm{x} 200$ ). (B) Cytoplasm of HGFs with positively stained for S100A4 (Rhodamine; magnification, x200). Vimentin and S100A4 are markers for mesenchymal cells DAPI is a marker of cell nuclei. HGF, human gingival fibroblast; DAPI, 4',6-diamidino-2-phenylindole.

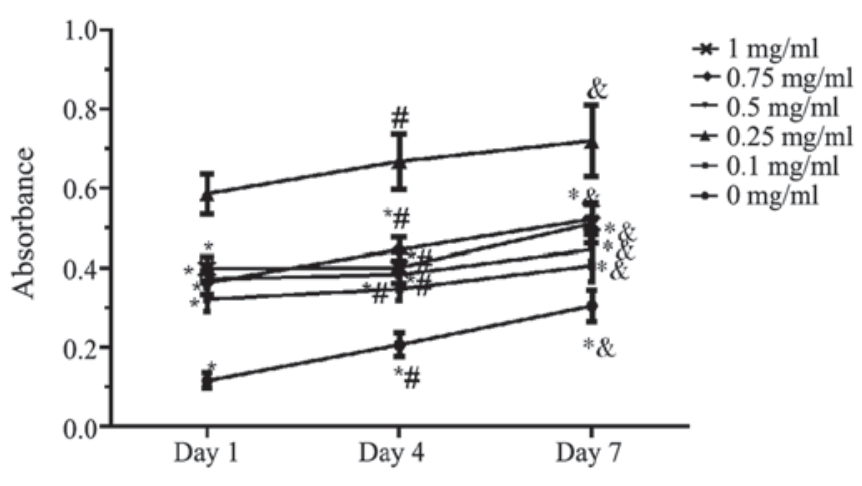

Figure 4. MTT assay. The cell adhesion ability of different concentrations of ACVM-HLC-I was evaluated by MTT assay on days 1, 4 and 7 ACVM-HLC-I, acellular vascular matrix-human-like collagen I. "P<0.05 vs. $0.25 \mathrm{mg} / \mathrm{ml},{ }^{\#} \mathrm{P}<0.05$ vs. day $1,{ }^{\&} \mathrm{P}<0.05$ vs. day 4 .

\section{Results}

General observations of the four scaffold materials. The ACVM-HLC-I scaffold presented as an ivory-white, translucent, non-elastic vascular wall with folded and damaged lumen (Fig. 1A). The ADM scaffold was ivory-white, translucent and exhibited a honeycomb structure with a rough surface (Fig. 1B). The thickness of ADM was 0.4-0.6 mm. Bio-Gide presented as pale yellow-white, translucent, rectangular patches with a smooth surface (Fig. 1C). SIS presented as a pale ivory, translucent membrane (Fig. 1D).

Observation of HGFs by inverted microscope. Following 7 days of primary culture of HGFs, fibroblast-like cells with clear and large outlines, spherical or elliptic nuclei were observed (Fig. 2A). At an average of 15 days, a large number of cells were available 

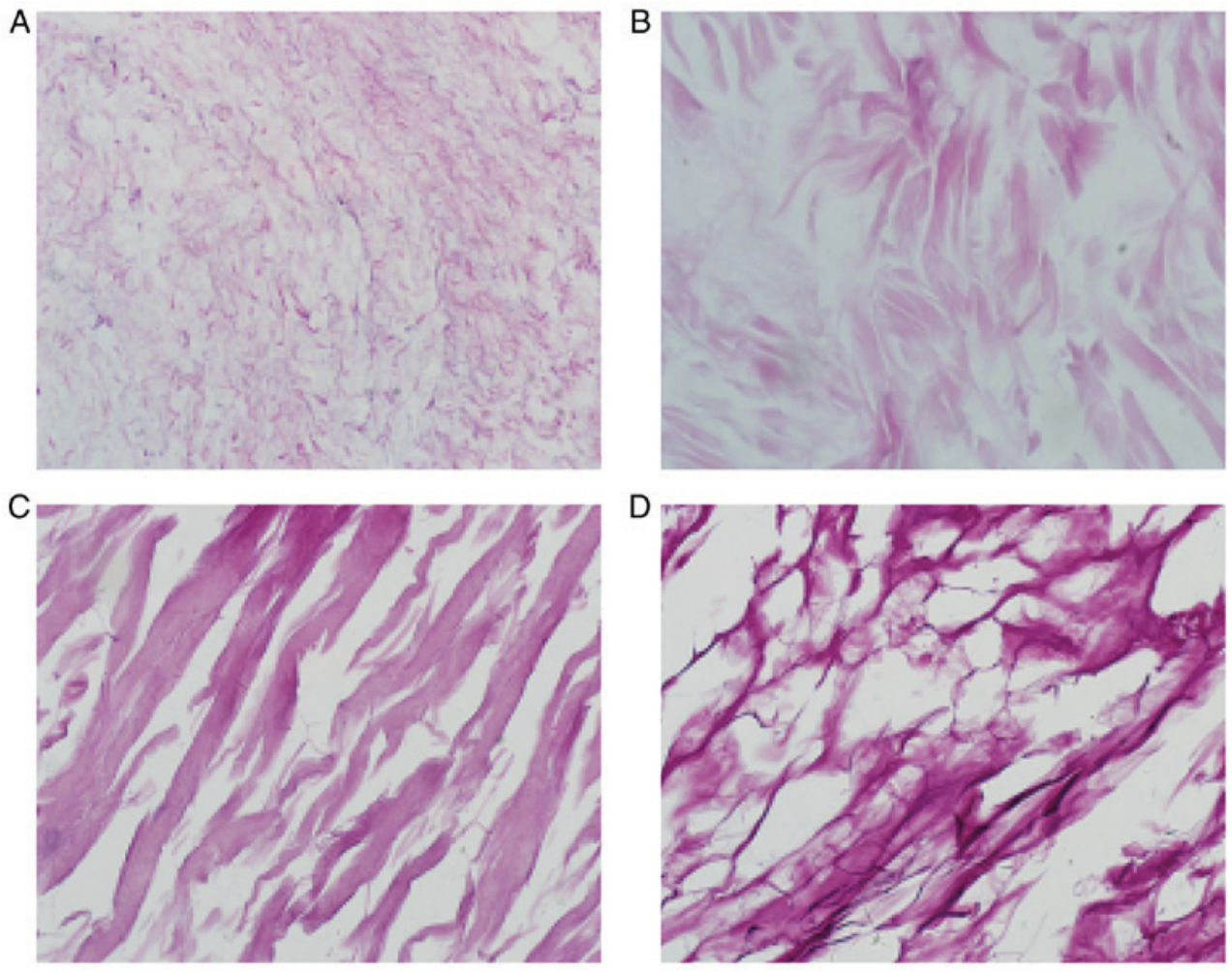

Figure 5. Hematoxylin and eosin staining of the scaffolds (magnification, x400). (A) ACVM-HLC-I, (B) ADM, (C) Bio-Gide and (D) SIS. ACVM-HLC-I contained red reticular fibers and collagen fibers. ADM exhibited a looser meshwork of collagen fibers, and Bio-Gide presented a looser meshwork of collagen fibers. SIS contained staggered and irregular collagen fibers. ACVM-HLC-I, acellular vascular matrix-human-like collagen I; ADM, acellular dermal matrix; SIS, small intestinal submucosa.
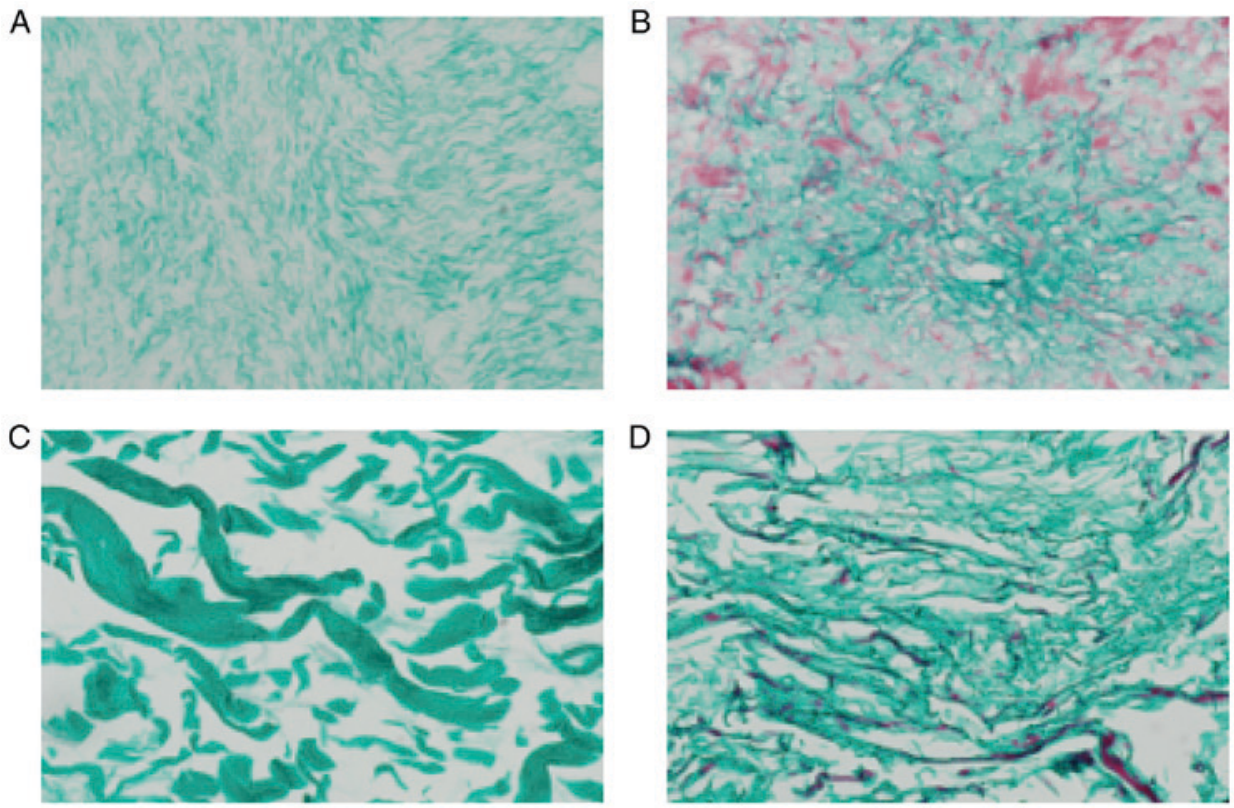

Figure 6. Masson staining of the scaffolds (magnification, x400). (A) ACVM-HLC-I, (B) ADM, (C) Bio-Gide and (D) SIS. Collagen fibers are stained green and muscle fibers are stained red. ACVM-HLC-I collagen fibers were soft and dense; the collagen fibers in each layer of ADM were dyed green, and muscle fibers were dyed red; the ADM collagen fiber structure was thin. The Bio-Gide collagen fibers were very thick and sparse. SIS consisted mostly of green collagen fibers and a small amount of red muscle fibers; the SIS collagen fibers were relatively sparse and hard. ACVM-HLC-I, acellular vascular matrix-human-like collagen I; ADM, acellular dermal matrix; SIS, small intestinal submucosa.

to harvest the fibroblast-like cells. As shown in (Fig. 2B), the second generation of cells was tightly adhered, spindle-shaped and well-spread, and fibroblast-like in appearance.
Identification of HGFs by immunofluorescent staining. The characteristics of HGFs were identified by immunofluorescent staining of vimentin and S100A4, markers for mesenchymal 

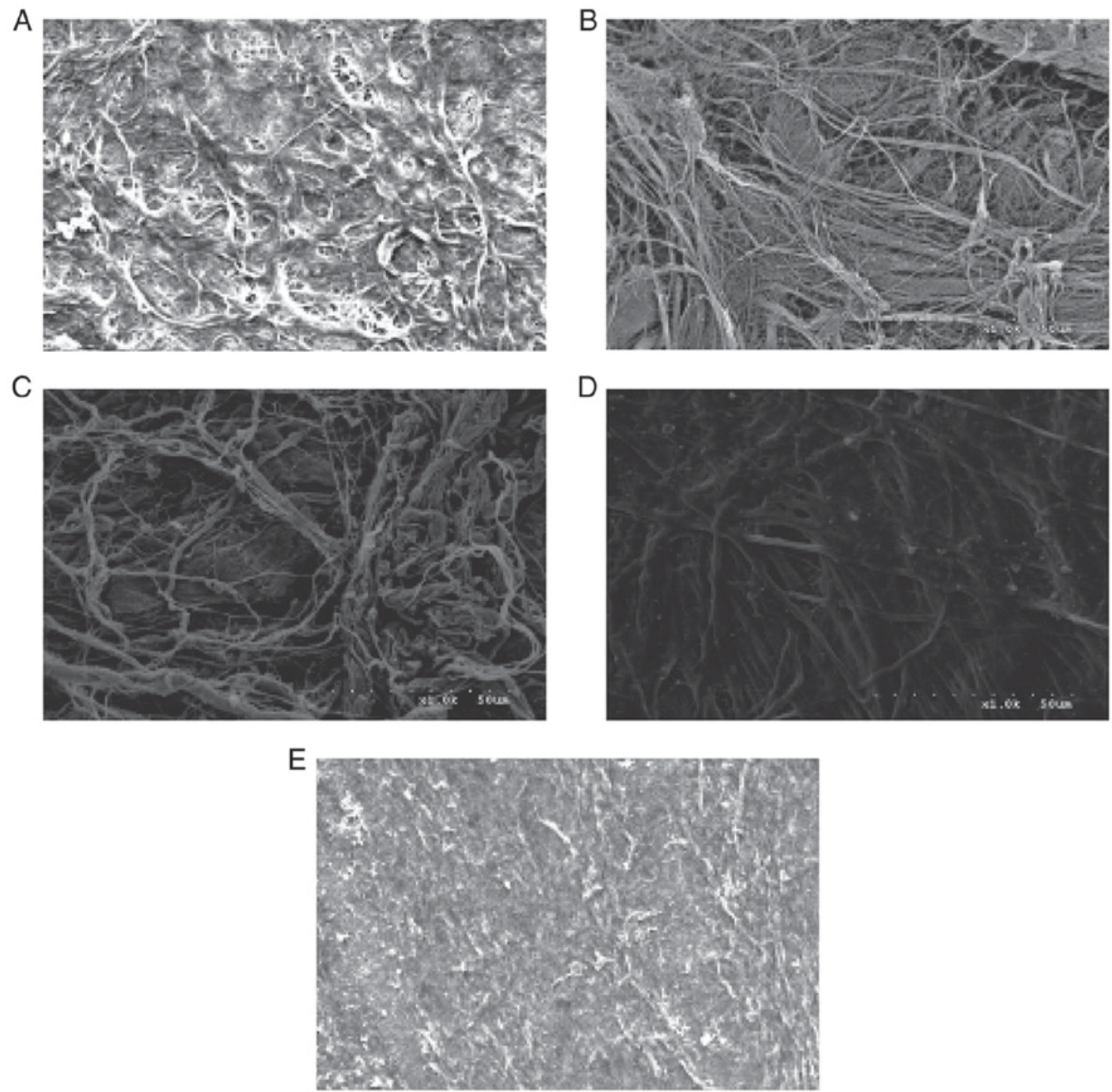

Figure 7. Scanning electron microscopy images of the scaffolds (magnification, x1,000). (A) ACVM, (B) ADM, (C) Bio-Gide, (D) SIS and (E) ACVM-0.25\% HLC-I. The surface fibers of the ACVM- $0.25 \%$ HLC-I scaffold were smaller and slender. ACVM, acellular vascular matrix; ACVM-0.25\% HLC-I, AVCM- $0.25 \%$ human-like collagen I; ADM, acellular dermal matrix; SIS, small intestinal submucosa.

cells. It was observed that the protein expression of vimentin and S100A4 was positive in the cytoplasm of HGFs (Fig. 3).

Cell adherence ability by MTT assay. The results demonstrated that compared with the ACVM scaffold without HLC-I, the ACVM-HLC-I scaffold had better cell adherence. In addition, the ACVM scaffold coated with $0.25 \%$ HLC-I presented the highest cell adherence at all time points (Fig. 4).

\section{Observation of vascular scaffolds}

$H \& E$ staining. H\&E staining of the ACVM- $0.25 \%$ HLC-I scaffold revealed the reticular and collagen fibers without cell nuclei, cell debris and media layer smooth muscle cells. The inner membrane was mainly distributed in the reticular fiber and media layer, the outer membrane was mainly distributed in the collagen fibers. The gap between collagen fibers was large, and collagen near the outer membrane was relatively concentrated (Fig. 5A). H\&E staining of the ADM scaffolds revealed that the mesh structure of collagen fibers was looser and fewer cells were present (Fig. 5B). H\&E staining of the
Bio-Gide scaffolds exhibited a loose and thick mesh structure of collagen fibers and complete absence of cells (Fig. 5C). In addition, it was observed that in the SIS scaffold, interlaced irregular collagen fibers produced a mesh with a large aperture and compact structure (Fig. 5D).

Masson staining. Masson staining of the four scaffolds suggested that the majority of them were composed of collagen fibers and were dyed green. In each layer of ADM, a large number of muscle fibers were dyed red, and the structure of the collagen fiber was thin. In the Bio-Gide group, the majority of the collagen fibers were dyed green, and the collagen fibers were thick and sparse. In the SIS scaffold only a small number of muscle fibers were dyed red (Fig. 6).

SEM. SEM images of the surfaces of the four scaffolds are presented in Fig. 7. The surfaces of the ADM, Bio-Gide, SIS and ACVM-0.25\% HLC-I scaffolds exhibited dense collagen fiber bundles. The material surface of the ACVM- $0.25 \%$ HLC-I scaffold was more slender and softer compared with 
those of the SIS, ADM and Bio-Gide scaffolds. In addition, compared with the Bio-Gide scaffold, the surface fiber structure of the ADM scaffold was dense, while the surface fiber structure of the SIS scaffold was relatively sparse and hard. The ACVM-0.25\% HLC-I scaffold surface appeared to have the smallest fibers, but no statistically significant difference was observed between SIS and ADM scaffolds.

Water absorption capacities of the scaffold materials. Following $24 \mathrm{~h}$, the ACVM-0.25\% HLC-I scaffold exhibited the best water absorption performance, followed by the SIS and Bio-Gide scaffolds, and then the ADM scaffold. The water absorption of the four scaffolds is presented in Table I. ACVM-0.25\% HLC-I scaffolds had significantly improved water absorption when compared with SIS, Bio-Gide and ADM scaffolds $(\mathrm{P}<0.05)$.

Mechanical test results of the scaffold materials. In terms of stress, the ADM scaffold material had the largest tensile stress and reached a peak value of $4.65 \pm 0.78 \mathrm{Mpa}$ at $46 \mathrm{sec}$, which was markedly higher when compared with the tensile stress of the ACVM-0.25\% HLC-I, Bio-Gide and SIS scaffold materials ( $\mathrm{P}<0.05$; Fig. 8). The tensile stress of ACVM- $0.25 \%$ HLC-I, Bio-Gide and SIS reached their peak values at 6, 16.8 and $22 \mathrm{sec}(0.48 \pm 0.04,0.52 \pm 0.09$ and $0.05 \pm 0.0017 \mathrm{Mpa})$, respectively. There was no notable difference in tensile stress between the ACVM-0.25\% HLC-I and Bio-Gide scaffold materials $(\mathrm{P}>0.05)$, but the tensile stress of the SIS scaffold material was markedly lower when compared with the other three types of scaffold $(\mathrm{P}<0.05)$. Therefore, the tensile stress of the ACVM- $0.25 \%$ HLC-I scaffold material was not as high as the ADM scaffold material, but was similar to the Bio-Gide scaffold material and was increased compared with the SIS scaffold material (Fig. 8).

In terms of strain, ADM exhibited a notably higher value compared with the ACVM-0.25\% HLC-I, Bio-Gide and SIS scaffold materials ( $\mathrm{P}<0.05$; Fig. 9). There was no notable difference between ACVM-0.25\% HLC-I and Bio-Gide scaffold materials $(\mathrm{P}>0.05)$, but there was a notable difference between ACVM-0.25\% HLC-I and SIS scaffold materials $(\mathrm{P}<0.05)$. The stress-strain curve indicated that the ADM scaffold had the greatest tensile strength. The tensile strength of the Bio-Gide scaffold was similar to that of the ACVM-0.25\% HLC-I scaffold, but the tensile strength of the SIS scaffold material was relatively poor (Figs. 8 and 9). In terms of breaking strength, the ADM scaffold material exhibited the highest strength, while the SIS scaffold material exhibited the lowest. There was no marked difference between the ACVM-0.25\% HLC-I and Bio-Gide scaffold materials $(\mathrm{P}>0.05)$. A negative association was observed between the elongation at break and the breaking strength (Table II). The largest value of elongation at break was observed in the SIS scaffold, while the smallest value was observed in the ADM scaffold. There was no notable difference in elongation at break between the Bio-Gide and ACVM-0.25\% HLC-I scaffolds (P>0.05; Table II).

\section{Observation of HGFs on vascular scaffolds}

HE staining. The histology of the ACVM-0.25\% HLC-I scaffold seeded with HGFs and cultured for 7 days is presented in Fig. 10. HGFs were abundant and well-distributed in the
Table I. Comparison of water absorption for four different biodegradable scaffolds.

\begin{tabular}{lcc}
\hline Scaffold & $\mathrm{n}$ & Water absorption $(\%)$ \\
\hline ACVM-0.25\% HLC-I & 6 & $24.05 \pm 0.59$ \\
SIS & 6 & $10.99 \pm 0.28$ \\
Bio-Gide & 6 & $5.82 \pm 0.07$ \\
ADM & 6 & $3.55 \pm 0.08$ \\
\hline
\end{tabular}

ACVM-0.25\% HLC-I, acellular vascular matrix-0.25\% human-like collagen I; ADM, acellular dermal matrix; SIS, small intestinal submucosa.

Table II. Breaking strength and elongation at break of the four scaffolds.

\begin{tabular}{lcc}
\hline Group & $\begin{array}{c}\text { Breaking } \\
\text { strength (MPa) }\end{array}$ & $\begin{array}{c}\text { Elongation } \\
\text { at break }(\%)\end{array}$ \\
\hline ADM & $46.5 \pm 1.78^{\mathrm{a}, \mathrm{b}}$ & $5.66 \pm 0.41^{\mathrm{a}, \mathrm{b}}$ \\
ACVM-0.25\% HLC-I & $4.80 \pm 0.32^{\mathrm{a}}$ & $52.93 \pm 1.34^{\mathrm{a}}$ \\
Bio-Gide & $5.20 \pm 0.35^{\mathrm{a}}$ & $51.31 \pm 1.48^{\mathrm{a}}$ \\
SIS & $0.50 \pm 0.08$ & $470.3 \pm 1.93$ \\
\hline
\end{tabular}

Data are presented as the mean \pm standard deviation. ${ }^{\mathrm{a}} \mathrm{P}<0.05$ vs. SIS group; ${ }^{\mathrm{P}}<0.05$ vs. Bio-Gide. ACVM-0.25\% HLC-I, acellular vascular matrix- $0.25 \%$ human-like collagen I; ADM, acellular dermal matrix; SIS, small intestinal submucosa.

central part of the ACVM-0.25\% HLC-I scaffold. The cells exhibited extending processes in various directions, indicating that they had potential migration abilities and cell activity. The histology of the HGFs seeded on the ADM and Bio-Gide scaffolds are also presented in Fig. 10. Compared with the ACVM-0.25\% HLC-I scaffold, ADM and Bio-Gide scaffolds following 7 days appeared to exhibit limited cell proliferation. The cells appeared healthy with extended morphology. The HGFs seeded on the SIS scaffold also presented and few cells grew on the surface of the SIS scaffold following 7 days (Fig. 10).

SEM. SEM images of the four scaffolds seeded with HGFs and cultured for 7 days are presented in Fig. 11. A large number of HGFs were observed on the surface of the ACVM- $0.25 \%$ HLC-I scaffold compared with the ADM and Bio-Gide scaffolds. On the other hand, HGFs were observed in limited numbers on the surface of the SIS scaffold. This result was similar to the histology results of the four different biodegradable scaffolds following seeding with HGFs.

Growth kinetics of HGFs on scaffolds. The number of HGFs in the ACVM-0.25\% HLC-I, SIS, ADM and Bio-Gide scaffolds were quantified by CCK- 8 assay (Fig. 12). The ACVM-0.25\% HLC-I scaffold exhibited a larger number of cells when compared with the other scaffolds. The ACVM-0.25\% HLC-I scaffold had the fastest level of 

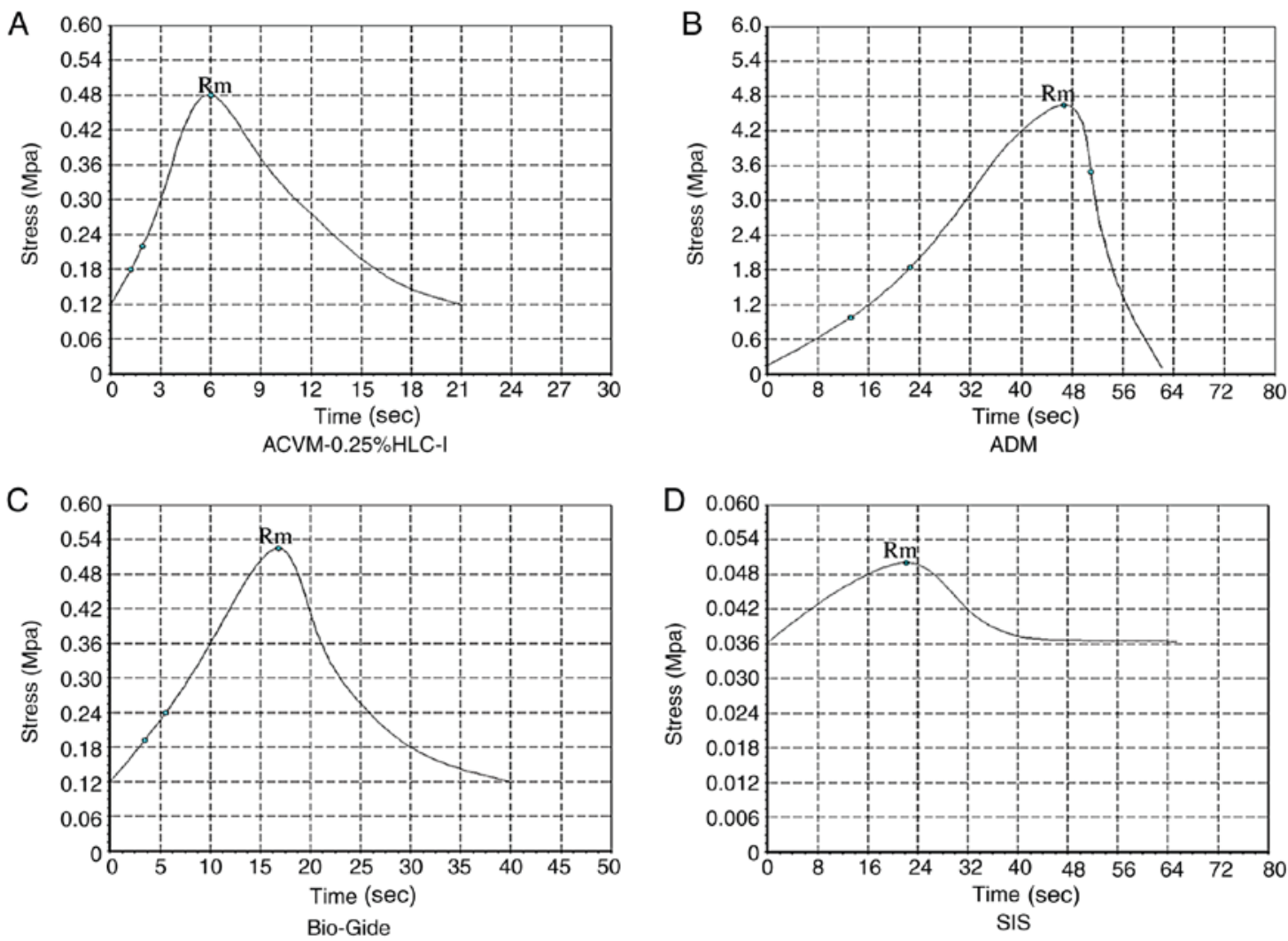

Figure 8. Stress-time curves of the scaffolds. (A) ACVM-0.25\% HLC-I, (B) ADM, (C) Bio-Gide and (D) SIS. The ADM scaffold exhibited the greatest tensile strength. ACVM-0.25\% HLC-I, acellular vascular matrix-0.25\% human-like collagen I; ADM, acellular dermal matrix; SIS, small intestinal submucosa; Rm, maximum pressure strength.
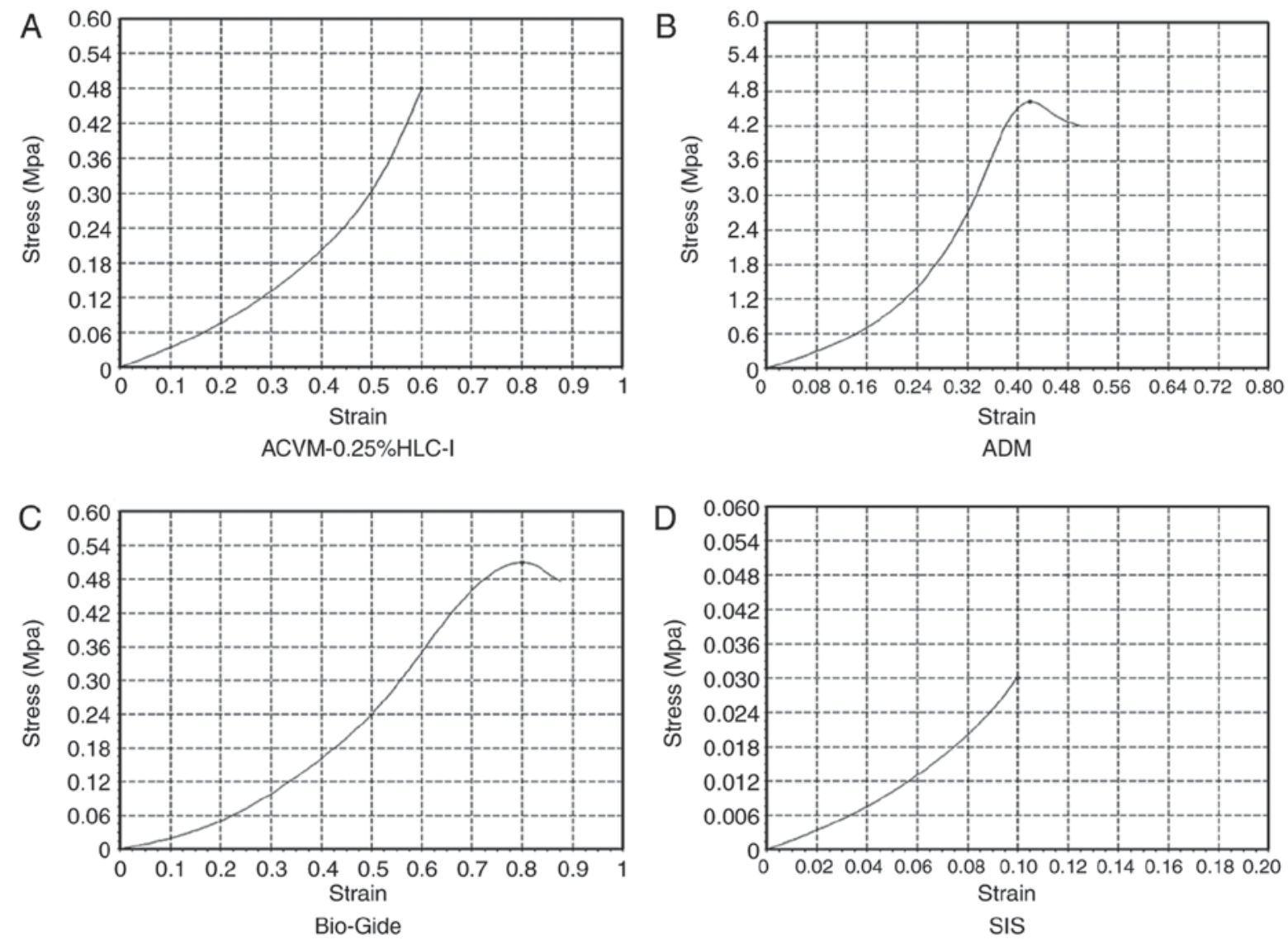

Figure 9. Stress-strain curves of the scaffolds. (A) ACVM-0.25\% HLC-I, (B) ADM, (C) Bio-Gide and (D) SIS. The ADM scaffold exhibited the greatest tensile strength. ACVM-0.25\% HLC-I, acellular vascular matrix-0.25\% human-like collagen I; ADM, acellular dermal matrix; SIS, small intestinal submucosa. 

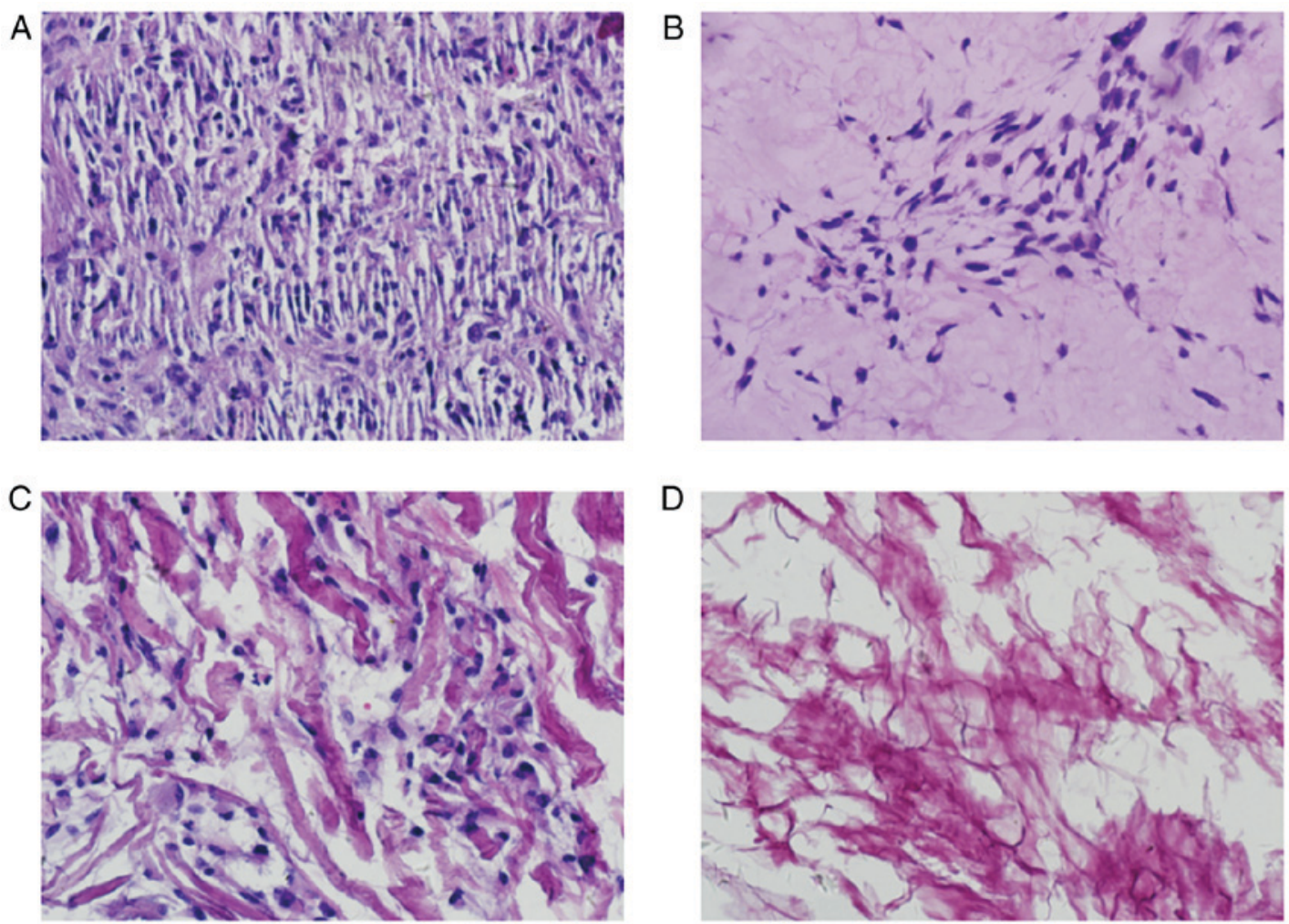

Figure 10. Hematoxylin and eosin staining indicating the histology of the scaffolds seeded with HGFs and cultured for 7 days (magnification, $\mathrm{x} 400$ ). (A) Cells-ACVM-0.25\% HLC-I, (B) Cells-ADM, (C) Cells-Bio-Gide and (D) Cells-SIS. HGFs were abundant and well-distributed in the central part of the ACVM- $0.25 \%$ HLC-I scaffold. The ADM and Bio-Gide scaffold presented reduced cell proliferation. Almost no cell proliferation was visible on the surface of the SIS scaffold. ACVM-0.25\% HLC-I, acellular vascular matrix-0.25\% human-like collagen I; ADM, acellular dermal matrix; SIS, small intestinal submucosa; HGF, human gingival fibroblast.

proliferation compared with the ADM, Bio-Gide and SIS scaffolds, with the majority of HGFs attaching following $1 \mathrm{~h}$ of incubation. There was no significant difference in the number of cells between the ADM and Bio-Gide scaffolds $(P>0.05)$. The number of cells in the SIS scaffold was lower when compared with the other scaffolds at all times. With the exception of the SIS scaffold, the number of cells collected from the scaffolds at $72 \mathrm{~h}$ was significantly increased from that at $24 \mathrm{~h}(\mathrm{P}<0.05$; Fig. 12).

Scaffold diameter prior to and following seeding with HGFs. The diameter of all scaffolds was $10 \mathrm{~mm}$ prior to seeding with HGFs. Following 7 days, the ACVM-0.25\% HLC-I scaffold had contracted significantly. The ADM and Bio-Gide scaffolds seeded with HGFs were slightly larger when compared with prior to seeding. There was no significant change in the SIS scaffold diameter following seeding with HGFs. The diameters of the four scaffolds prior to and following seeding with HGFs are presented in Table III.

\section{Discussion}

The most widely used scaffold materials in tissue engineering research are natural biological scaffold materials, synthetic degradable polymer scaffolds and composite scaffold materials. In the present study, the SIS $(28,29)$ and ADM scaffolds were used as natural biological scaffold materials, and the Bio-Gide scaffold material was a synthetic degradable polymer. The ACVM-HLC-I scaffold combined ACVM material with HLC-I to form a composite scaffold material. Previous research has indicated that composite scaffold materials possess the good cellular compatibility of natural biological scaffolds and the strong mechanical properties of synthetic degradable polymer scaffolds (31). In the present study, ACVM-HLC-I was compared with ADM, Bio-Gide and SIS, and the aim of the study was to verify the feasibility of the composite scaffold material ACVM-HLC-I for application as a tissue engineering scaffold material. Preliminary findings had confirmed that the ACVM-HLC-I composite scaffold material was feasible for the construction of tissue-engineered blood vessels. ACVM- $0.25 \%$ HLC-I provides a three-dimensional ultrastructure space for the growth of seeded cells and promotes the establishment of an organized structure and function (32). It also has appropriate mechanical properties for maintaining compressive resistance, which is required for building blood vessel substitutes. A concentration of $0.25 \%$ HLC-I was demonstrated to be optimal (23). The preparation of ACVM- $0.25 \%$ HLC-I material supported the tissue engineering of blood vessels by inducing vascular endothelial cells and smooth muscle cells (33). In the present study, the application feasibility of composite scaffold material ACVM- $0.25 \%$ HLC-I was explored further and compared with other tissue engineering scaffold materials, ADM, Bio-Gide and SIS.

In the present study, the ADM material was a transparent, milky-yellow film 0.4-0.6 mm thick, which was divided into a coarse hairy surface and a translucent honeycomb smooth 
A

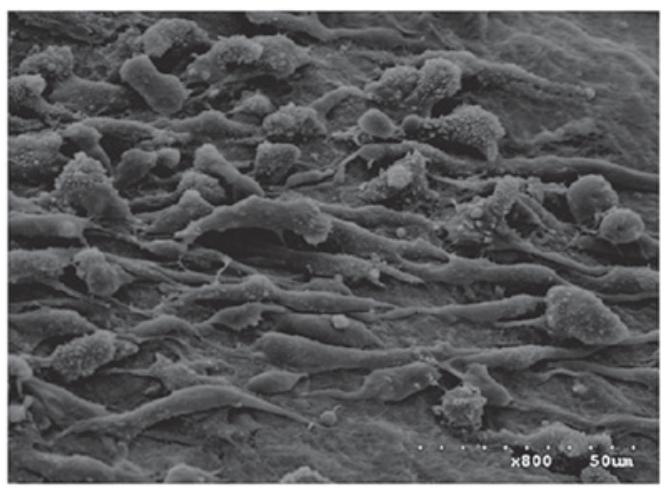

$\mathrm{C}$

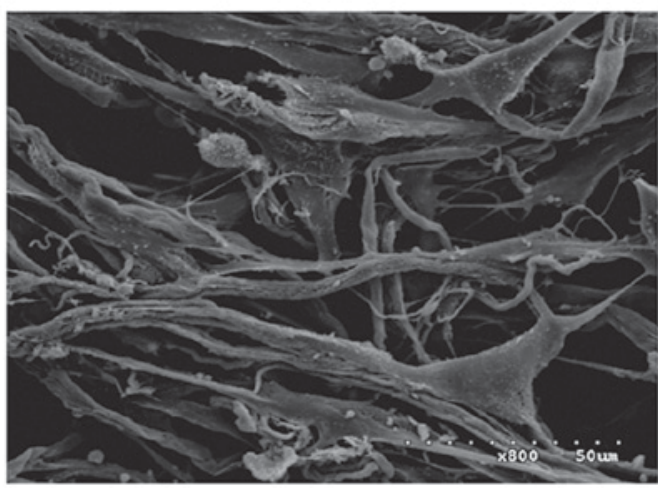

$\mathrm{B}$

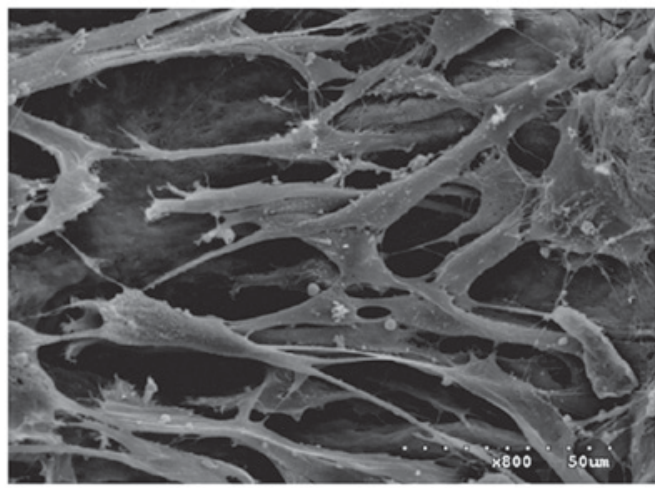

D

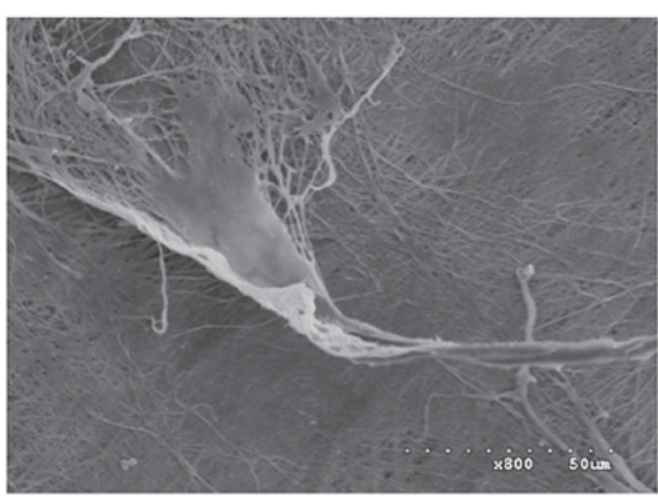

Figure 11. Scanning electron microscopy images of the four scaffolds seeded with HGFs and cultured for 7 days (magnification, $\mathrm{x} 800$ ). (A) Cells-ACVM-0.25\% HLC-I, (B) Cells-ADM, (C) Cells-Bio-Gide and (D) Cells-SIS. A large number of HGFs were visible on the surface of the ACVM-0.25\% HLC-I scaffold. The SIS scaffold had the smallest number of HGFs on the surface. ACVM-0.25\% HLC-I, acellular vascular matrix- $0.25 \%$ human-like collagen I; ADM, acellular dermal matrix; SIS, small intestinal submucosa; HGF, human gingival fibroblast.

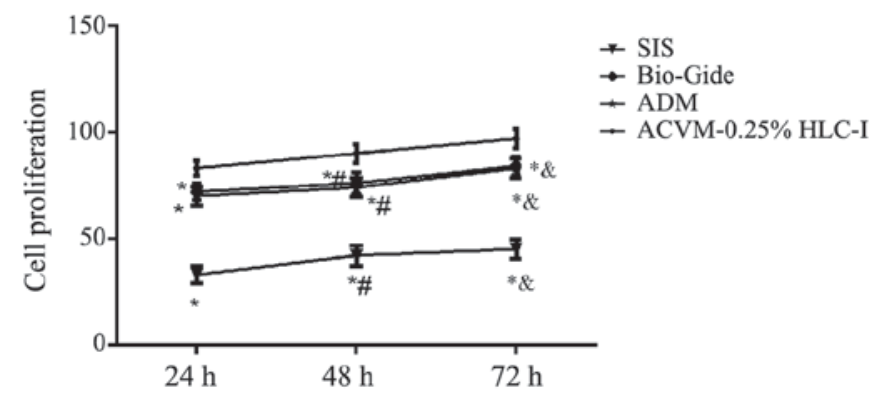

Figure 12. Number of HGFs in the scaffolds as determined by Cell Counting Kit-8 assay. A large number of proliferative HGFs was observed in the ACVM- $0.25 \%$ HLC-I scaffold. There is no significant difference in the number of cells between ADM and Bio-Gide scaffolds. The lowest number of proliferative HGFs was observed in the SIS scaffold. ACVM-0.25\% HLC-I, acellular vascular matrix-0.25\% human-like collagen I; ADM, acellular dermal matrix; SIS, small intestinal submucosa; HGF, human gingival fibroblast. ${ }^{*} \mathrm{P}<0.05$ vs. ACVM-0.25\% HLC-I, ${ }^{\#} \mathrm{P}<0.05$ vs. $24 \mathrm{~h},{ }^{\circledR} \mathrm{P}<0.05$ vs. $48 \mathrm{~h}$.

surface with slightly elastic force. The Bio-Gide material was a white membrane, which was slightly less thick than the ADM scaffold material. Large collagen bundles could be seen protruding from the surface of the Bio-Gide scaffold, and the elasticity was low. The SIS scaffold material was a yellow and white translucent membrane with low elasticity. The ACVM- $0.25 \%$ HLC-I scaffold was a homogeneous, translucent membrane with low thickness and high elasticity. H\&E staining, Masson staining and SEM indicated that there were dense bundles of collagen fibers on the surface of the ADM,
Bio-Gide, SIS and ACVM- $0.25 \%$ HLC-I scaffolds, but on the ACVM- $0.25 \%$ HLC-I scaffold these were more slender and soft compared with those on the SIS, ADM and Bio-Gide scaffolds. In addition, compared with the Bio-Gide scaffold, the surface fiber structure of the ADM scaffold was dense, while the surface fiber structure of the SIS scaffold was relatively sparse and hard.

Previous studies have indicated that scaffold materials applied in tissue engineering should have a degree of biomechanical strength $(23,30)$, also called mechanical strength, to ensure that they do not become deformed or ruptured when implanted in the body of a human or animal. For example, tissue-engineered blood vessels should have a certain amount of compressive stress and shear stress to tolerate blood flow impact without breaking. To compare the strength of ADM, Bio-Gide, SIS and AVCM-0.25\% HLC-I materials in the present study, biomechanical test apparatus was used for various mechanical experiments. In terms of stress, ADM scaffold materials reached a maximum tensile stress of $4.65 \pm 0.78 \mathrm{Mpa}$ at $46 \mathrm{sec}$, which was significantly higher compared with the ACVM-0.25\% HLC-I, Bio-Gide and SIS scaffold materials. Among the groups, there was no significant difference in tensile stress between the ACVM-0.25\% HLC-I and Bio-Gide scaffold materials, but the SIS scaffold material had significantly lower tensile stress compared with other three scaffold materials. There was a significant difference in strain among the ADM, ACVM- $0.25 \%$ HLC-I, Bio-Gide and SIS scaffold materials. The ACVM- $0.25 \%$ HLC-I was not significantly different compared with the Bio-Gide scaffold, but was significantly different compared with the SIS scaffold. 
Table III. Diameter of the four different biodegradable scaffolds before and after seeding with HGFs.

\begin{tabular}{lcc}
\hline & \multicolumn{2}{c}{ Diameter of scaffold (mm) } \\
\cline { 2 - 3 } Scaffold & $\begin{array}{l}\text { Prior to } \\
\text { seeding }\end{array}$ & $\begin{array}{r}\text { Following HGF } \\
\text { seeding for 7 days }\end{array}$ \\
\hline ACVM-0.25\% HLC-I & 10.00 & $9.77^{\mathrm{a}}$ \\
SIS & 10.00 & 10.00 \\
Bio-Gide & 10.00 & 10.02 \\
ADM & 10.00 & 10.04 \\
\hline
\end{tabular}

ACVM-0.25\% HLC-I, acellular vascular matrix-0.25\% human-like collagen I; ADM, acellular dermal matrix; SIS, small intestinal submucosa; HGF, human gingival fibroblast. ${ }^{a} \mathrm{P}<0.05$ vs. prior to seeding.

A stress-strain curve was constructed for the ADM, Bio-Gide, SIS and ACVM-0.25\% HLC-I scaffold materials. The ADM scaffold had the largest tensile capacity. The resistance of the Bio-Gide scaffold was similar to that of the ACVM- $0.25 \%$ HLC-I, while the tensile strength of the SIS scaffold material was relatively poor. According to the fracture strength value, ADM scaffolds had the highest fracture strength. The ACVM- $0.25 \%$ HLC-I scaffold and Bio-Gide material had a similar fracture strength and were weaker compared with the ADM scaffold. The SIS scaffold had the lowest fracture strength. The elongation at break value was highest for the SIS scaffold, followed by the Bio-Gide and ACVM- $0.25 \%$ HLC-I scaffolds. The ADM scaffold had the lowest elongation at break value.

The aforementioned results indicated that the breaking strength values of the ADM, Bio-Gide, ACVM- $0.25 \%$ HLC-I and SIS scaffolds were negatively associated with elongation at break. Although ADM scaffolds had the highest breaking strength, their elongation at break was the smallest. Although SIS scaffolds had the smallest breaking strength, their elongation at break was the largest. There was no significant difference between the Bio-Gide and ACVM-0.25\% HLC-I scaffolds in terms of breaking strength and elongation at break. This suggested that although the tensile stress, stress strain and breaking strength of the ACVM- $0.25 \%$ HLC-I scaffold were not as large as that of the ADM scaffold material, it had similar properties to the Bio-Gide scaffold material. As composite scaffolds, ACVM-0.25\% HLC-I scaffolds have similar mechanical properties and mechanical strength to synthetic biodegradable polymer scaffolds; that is, ACVM- $0.25 \%$ HLC-I scaffolds have appropriate mechanical strength and other mechanical properties to act as scaffolds for tissue engineering.

The results of the water absorption test indicated that ACVM- $0.25 \%$ HLC-I scaffolds had a significantly greater water absorption capacity compared with the SIS, Bio-Gide and ADM scaffolds. The SIS scaffold had the worst mechanical properties, but its water absorption capacity was only inferior to that of the ACVM- $0.25 \%$ HLC-I scaffold. Although the mechanical capacity of Bio-Gide scaffold was not significantly different to that of the ACVM-0.25\% HLC-I scaffold, its water absorption ability was markedly lower compared with the ACVM-0.25\% HLC-I scaffold. The mechanical strength of the ADM scaffold was the largest, but its water absorption capacity was the poorest. Based on the aforementioned experimental results, it was concluded that the ACVM-0.25\% HLC-I scaffold possessed biomechanical properties and a strong water absorption capability, further indicating its feasibility as a tissue engineering scaffold material.

Furthermore, scaffold materials for tissue engineering should have no cytotoxic effects in addition to possessing biomechanical strength (34). The results indicated that the cytotoxicity of ACVM- $0.25 \%$ HLC-I scaffolds was significantly different from that of the ADM, Bio-Gide and SIS scaffolds, while there was no significant difference in cytotoxicity between ADM and Bio-Gide scaffolds. The relative growth rate (RGR) of ACVM- $0.25 \%$ HLC-I, ADM and Bio-Gide scaffolds was $>75 \%$ at 24,48 and $72 \mathrm{~h}$, the cytotoxicity grade was 1 , and the scaffolds were considered to not be cytotoxic. The RGR of the SIS scaffolds was $>40 \%$ at $24 \mathrm{~h}$, and the scaffolds were considered to be cytotoxic. The RGR was $>50 \%$ at 48 and $72 \mathrm{~h}$, and the scaffolds were considered to not be cytotoxic. This suggested that, as a scaffold for tissue engineering, the ACVM- $0.25 \%$ HLC-I scaffold had improved cellular compatibility and lower cytotoxicity compared with the ADM, Bio-Gide and SIS scaffolds, which provides a basis for screening of the material in vivo implants. Subsequently, an experiment was conducted to verify whether the diameter of the material changed following seeding with HGFs. The results indicated that following seeding with HGFs, the diameter of the ACVM- $0.25 \%$ HLC-I scaffold decreased and the diameter of the SIS scaffold had not markedly changed. The diameters of the ADM and Bio-Gide scaffolds increased slightly. This may be associated with good attachment and growth of HGFs on the ACVM- $0.25 \%$ HLC-I scaffold.

ACVM- $0.25 \%$ HLC-I scaffold material, which combines HLC-I $(23)$ with ACVM $(23,24)$, is a composite tissue engineering scaffold formed by combining natural acellular biomaterials with synthetic polymer materials. Composite materials have the advantages of natural acellular biomaterials (such as ADM scaffolds) and synthetic polymer materials (such as Bio-Gide scaffolds). Therefore, they can provide three-dimensional ultrastructural protein space for cell growth and promote the formation of tissue function. They also possess sufficient mechanical properties to maintain the compressive ability of the tissue substitutes and the degradation rate of the material, and can be designed and regulated $(35,36)$. In conclusion, the results of the present study indicate that ACVM- $0.25 \%$ HLC-I promotes cell adhesion, cell proliferation and cell migration, and possesses good mechanical and biomechanical properties. This suggests that the composite tissue engineering scaffold material could be successfully applied in tissue regeneration.

The limitations in the present study must also be mentioned. Firstly, during the experiment, the authors did not consider to evaluate the potential change of the scaffold material following 7 days in mechanical properties, only testing the mechanical properties of the scaffold material prior to planting the cells. Secondly, no in vivo tests were performed on animals, which was a flaw in our experimental design, thus this will be addressed in future studies. 


\section{Acknowledgements}

Not applicable.

\section{Funding}

The present study was supported by Applied basic research found of Luzhou Science and Technology Bureau of Sichuan province (grant no. 2018-JYJ-36); City-School-Stomatological Hospital Introduces Doctoral Research Fund (grant no. 20186-2); Scientific Research Fund of Sichuan Medical Association (grant no. S17073)

\section{Availability of data and materials}

All data generated or analyzed during the present study are included in this article.

\section{Authors' contributions}

All authors conceived and designed the experiments. YLQ, $\mathrm{XC}$ and YLH performed the experiments and analyzed the data. YJH, SBT and YHC wrote the manuscript. LY, MHN and $\mathrm{XQL}$ modified the manuscript and designed the experiments.

\section{Ethics approval and consent to participate}

Animal experiments were approved by the Ethics Committee of Southwest Medical University (Sichuan, China). The acquisition of normal human gingival tissues from patients was approved by the Hospital of Stomatology at Hebei Medical University (Hebei, China), and written informed consent was obtained from all patients.

\section{Patient consent for publication}

Not applicable.

\section{Competing interests}

The authors declare that they have no competing interests.

\section{References}

1. Gao P, Jiang D and Li Z: The application progress of human urine derived stem cells in bone tissue engineering. Zhonghua Wai Ke Za Zhi 54: 317-320, 2016 (In Chinese).

2. Sun $Z$ and Li J: Research progress of tissue engineered ligament. Zhongguo Xiu Fu Chong Jian Wai Ke Za Zhi 29: 1160-1166, 2015 (In Chinese).

3. Yang Q, Xu HW, Hurday S and Xu BS: Construction strategy and progress of whole intervertebral disc tissue engineering. Orthop Surg 8: 11-18, 2016.

4. Ge L, Cao C, Chen S, Shao B, Li Q, Li Q, Liu L, Liang Z and Huang Y: Preparation of laminin/nidogen adsorbed urinary bladder decellularized materials via a mussel-inspired polydopamine coating for pelvic reconstruction. Am J Transl Res 9: 5289-5298, 2017

5. Mi HY,Jing X, Napiwocki BN, Hagerty BS, Chen G and Turng LS: Biocompatible, degradable thermoplastic polyurethane based on polycaprolactone-block-polytetrahydrofuran-block-polycaprolactone copolymers for soft tissue engineering. J Mater Chem B 5: 4137-4151, 2017.

6. Wang L, Yang J, Ran B, Yang X, Zheng W, Long Y and Jiang X: Small molecular TGF- $\beta 1$-inhibitor-loaded electrospun fibrous scaffolds for preventing hypertrophic scars. ACS Appl Mater Interfaces 9: 32545-32553, 2017.
7. Faulk DM, Londono R, Wolf MT, Ranallo CA, Carruthers CA, Wildemann JD, Dearth CL and Badylak SF: ECM hydrogel coating mitigates the chronic inflammatory response to polypropylene mesh. Biomaterials 35: 8585-8595, 2014.

8. Wang W, Deng D, Wang B, Zhou G, Zhang W, Cao Y, Zhang P and Liu W: * Comparison of autologous, allogeneic, and cell-free scaffold approaches for engineered tendon repair in a rabbit model-a pilot study. Tissue Eng Part A 23: 750-761, 2017.

9. Xie J, Peng C, Zhao Q, Wang X, Yuan H, Yang L, Li K, Lou X and Zhang Y: Osteogenic differentiation and bone regeneration of iPSC-MSCs supported by a biomimetic nanofibrous scaffold. Acta Biomater 29: 365-379, 2016.

10. Baumgartner W, Schneider I, Hess SC, Stark WJ, Märsmann S, Brunelli M, Calcagni M, Cinelli P and Buschmann J: Cyclic uniaxial compression of human stem cells seeded on a bone biomimetic nanocomposite decreases anti-osteogenic commitment evoked by shear stress. J Mech Behav Biomed Mater 83: 84-93, 2018.

11. Qiu XW and Wang JH: Efficacy of dermal scaffold for promoting repair of acute full-thickness skin defects in pigs. Nan Fang Yi Ke Da Xue Xue Bao 38: 363-368, 2018 (In Chinese).

12. Ramanathan G, MuthukumarT and Tirichurapalli Sivagnanam U: In vivo efficiency of the collagen coated nanofibrous scaffold and their effect on growth factors and pro-inflammatory cytokines in wound healing. Eur J Pharmacol 814: 45-55, 2017.

13. Farkas B, Rodio M, Romano I, Diaspro A, Intartaglia R and Beke S: Fabrication of hybrid nanocomposite scaffolds by incorporating ligand-free hydroxyapatite nanoparticles into biodegradable polymer scaffolds and release studies. Beilstein J Nanotechnol 6: 2217-2223, 2015.

14. Nasonova MV, Glushkova TV, Borisov VV, Velikanova EA, Burago AY and Kudryavtseva YA: Biocompatibility and structural features of biodegradable polymer scaffolds. Bull Exp Biol Med 160: 134-140, 2015.

15. Perry L, Flugelman MY and Levenberg S: Elderly patient-derived endothelial cells for vascularization of engineered muscle. Mol Ther 25: 935-948, 2017.

16. Hu WW, Wu YC and Hu ZC: The development of an alginate/polycaprolactone composite scaffold for in situ transfection application. Carbohydr Polym 183: 29-36, 2018.

17. Sun T, Liu M, Yao S, Ji Y, Xiong Z, Tang K, Chen K, Yang H and Guo XD: Biomimetic composite scaffold containing small intestinal submucosa and mesoporous bioactive glass exhibits high osteogenic and angiogenic capacity. Tissue Eng Part A 24: 1044-1056, 2018.

18. Tohamy KM, Mabrouk M, Soliman IE, Beherei HH and Aboelnasr MA: Novel alginate/hydroxyethyl cellulose/hydroxyapatite composite scaffold for bone regeneration: In vitro cell viability and proliferation of human mesenchymal stem cells. Int J Biol Macromol 112: 448-460, 2018.

19. Awad NK, Niu H, Ali U, Morsi YS and Lin T, et al: Electrospun fibrous scaffolds for small-diameter blood vessels: A review. Membranes (Basel) 8: E15, 2018.

20. Xu Y, Wu J, Wang H, Li H, Di N, Song L, Li S, Li D, Xiang Y, Liu W, et al: Fabrication of electrospun poly(L-lactide-co- $\varepsilon$-cap rolactone)/collagen nanoyarn network as a novel, three-dimensional, macroporous, aligned scaffold for tendon tissue engineering. Tissue Eng Part C Methods 19: 925-936, 2013.

21. Feng C, Hu J, Liu C, Liu S, Liao G, Song L and Zeng X: Association of 17- $\beta$ estradiol with adipose-derived stem cells: New strategy to produce functional myogenic differentiated cells with a nano-scaffold for tissue engineering. PLoS One 11: e0164918, 2016.

22. Lech CJ and Phan AT: Ball with hair: Modular functionalization of highly stable G-quadruplex DNA nano-scaffolds through N2-guanine modification. Nucleic Acids Res 45: 6265-6274, 2017.

23. Liu X, Wang J, Dong F, Song P, Tian S, Li H and Hou Y: Cytocompatibility and biologic characteristics of synthetic scaffold materials of rabbit acellular vascular matrix combining with human-like collagen I. J Biomater Appl 32: 463-471, 2017.

24. Lalka SG, Oelker LM, Malone JM, Duhamel RC, Kevorkian MA, Raper BA, Nixon JC, Etchberger KJ, Dalsing MC, Cikrit DF, et al: Acellular vascular matrix: A natural endothelial cell substrate. Ann Vasc Surg 3: 108-117, 1989.

25. Liu X, Wang J, Dong F, Song P, Li H and Hou Y: Study of composite vascular scaffold combining with differentiated VSMC- and VEC-like cells in vitro and in vivo. J Biomater Appl 32: 219-229, 2017.

26. Heidemann LN, Gunnarsson GL, Salzberg CA, Sørensen JA and Thomsen JB: Complications following nipple-sparing mastectomy and immediate acellular dermal matrix implant-based breast reconstruction-a systematic review and meta-analysis. Plast Reconstr Surg Glob Open 6: e1625, 2018. 
27. Sobti N, JiE, Brown RL, Cetrulo CL Jr, Colwell AS, Winograd JM Austen WG Jr and Liao EC: Evaluation of acellular dermal matrix efficacy in prosthesis-based breast reconstruction. Plast Reconstr Sur 141: 541-549, 2018

28. Pitman MJ, Kurita T, Powell ME, Kimball EE, Mizuta M, Chang S, Garrett CG and Rousseau B: Vibratory function and healing outcomes after small intestinal submucosa biomaterial implantation for chronic vocal fold scar. Laryngoscope 128: 901-908, 2018

29. Zhou Q, Teng F, Zhang Y, Sun Q and Meng G: Evaluation of transventricular placement of porcine small intestinal submucosa stent valves in the pulmonary position in juvenile sheep model. Interact Cardiovasc Thorac Surg 27: 295-300, 2018.

30. Liu X, Wang J, Dong F, Li H and Hou Y: Induced differentiation of human gingival fibroblasts into VSMC-like cells. Differentiation 95: 1-9, 2017.

31. Choi HJ, Lee JJ, Lee JB, Sung HJ, Shin JW, Shin JW, Wu Y and Kim JK: MG-63 cells proliferation following various types of mechanical stimulation on cells by auxetic hybrid scaffolds. Biomater Res 20: 32, 2016.

32. Zhang W, Choi JK and He X: Engineering microvascularized 3D tissue using alginate-chitosan microcapsules. J Biomater Tissue Eng 7: 170-173, 2017.
33. Wang Y, Yin P, Bian GL, Huang HY, Shen H, Yang JJ, Yang ZY and Shen ZY: The combination of stem cells and tissue engineering: An advanced strategy for blood vessels regeneration and vascular disease treatment. Stem Cell Res Ther 8: 194, 2017.

34. Saberi EA, Karkehabadi H and Mollashahi NF: Cytotoxicity of various endodontic materials on stem cells of human apical papilla. Iran Endod J 11: 17-22, 2016.

35. Wu T, Huang C,LiD, Yin A, Liu W, Wang J,Chen J,Ei-Hamshary H, Al-Deyab SS and Mo X: A multi-layered vascular scaffold with symmetrical structure by bi-directional gradient electrospinning. Colloids Surf B Biointerfaces 133: 179-188, 2015.

36. Jiang B, Akar B, Waller TM, Larson JC, Appel AA and Brey EM: Design of a composite biomaterial system for tissue engineering applications. Acta Biomater 10: 1177-1186, 2014.

This work is licensed under a Creative Commons Attribution-NonCommercial-NoDerivatives 4.0 International (CC BY-NC-ND 4.0) License. 